\title{
THE EFFECTS OF STORAGE TEMPERATURE AND TYPES OF MEDIA ON THE SURVIVAL RATE OF S. agalactiae BACTERIOPHAGE
}

\author{
Angela Mariana Lusiastuti*), Tuti Sumiati**), and Adin Priadi***)
}

\begin{abstract}
The successful effect of phages in phage therapy has been proven by an increase of the presence of phages in survivors. Seven previously isolated phages were used to examine the effects of storage temperature and types of media on their survival rate. Phages PSaT-18, PSaT-19, PSaT-20 and PSaT-21 isolated from a Streptococcosis-infected Tilapia (Oreochromis niloticus) were used in this study. Phages PSaG-1, PSaG-2 and PSaG-3 were isolated from a diseased Gouramy (Osphronemus gouramy). All of the phages were stored in refrigerator at temperature of $8^{\circ} \mathrm{C}$ and kept in two types of media, Brain Heart Infusion Broth (BHIB) and BHIB enriched with glycerol. To determine the lytic activity of the phages, $20 \mu \mathrm{L}$ of each phage suspension was poured onto BHIA plate and incubated at $25^{\circ} \mathrm{C}$ for 24 to 48 hours. The result showed that phages PSaT-18, PSaT-19, PSaT-20 and PSaT-21 have lytic activity against $S$. agalactiae and $S$. iniae than phages PSaG-1, PSaG-2, and PSaG3. Phages PSaT-18, PSaT-19, PSaT-20, and PSaT-21 always survived up to three weeks and phage PSaT-1 8 in BHIB have the highest potential as a candidate of phage therapy for Streptococcosis.
\end{abstract}

KEYWORDS: storage temperature, types of media, survival rate, bacteriophage

\section{INTRODUCTION}

Bacterial resistance to antibiotics has become a serious health problem in fish. Ironically, resistance is promoted by both the overuse of antibiotics as well as insufficiency of dose. A possible alternative for the treatment of bacterial infections could be the use of bacteriophages, viruses that live on bacteria. Theoretically, bacteriophages can be used to treat infectious disease, but little attention has been focused to the phage therapy and prophylaxis in fish. However, a series of successful phage therapies for human bacterial infections and experimental animals was described by Slopek et al. (1987).

Compared with chemical antibiotics, bacteriophages offer several advantages: (1) lim- ited impact. Unlike antibiotics, bacteriophages are self-replicating as well as self-limiting. Bacteriophages replicate exponentially as long as the specific bacteria are available in abundance; (2) limited resistance development, phages can out compete the adaptation of the bacteria, and development of resistance is therefore limited; (3) specific targets, bacteriophages specify their targets to a particular kind of bacteria compared to chemical antibiotics, and therefore cause much less damage to the human intestinal microorganism(Lorch, 1999; Nakai \& Park, 2002).

Streptococcus agalactiae is the causative agent of Streptococcosis in farmed fish worldwide. As S. agalactiae is ubiquitous in fish and their culture environments, it is believed that

\footnotetext{
"Research Institute for Freshwater Aquaculture, Bogor, Indonesia

**) Central Research Institute for Aquaculture, Jakarta, Indonesia

${ }^{* * *}$ Research Institute for Veteriner, Bogor, Indonesia
} 
the bacterium is a typical opportunistic pathogen, and therefore reducing stress factors, such as poor water quality, overcrowding, overfeeding and insufficient nutrition is generally important in controlling the disease (Plumb, 1994). The economic losses caused by Streptococcosis in aquaculture farms worldwide are estimated to be over US $\$ 100$ million per year (Shoemaker et al., 2001). In this research, we evaluated the effects of storage temperature and types of media on the survival rate of $S$. agalactiae bacteriophages. The benefit of this research was to find the best bacteriophage as a candidate on phage therapy for Streptococcosis to replace the antibiotic therapy.

\section{METHODS}

Clinically isolates of $S$. agalactiae and $S$. iniae were isolated from brain, kidney and liver of diseased Tilapia. All bacterial strains were grown in Brain Heart Infusion Agar plates (BHIA, Difco) for 24 to 48 hours at $28^{\circ} \mathrm{C}$. The predominant types of colonies were subcultured and subjected to bio-chemical and physiological tests including catalase production, haemolytic activity in blood agar, and test of API STREP 20 System. Confirmatory identification was performed by PCR.

Seven phages designated as PSaT-18, PSaT-1 9, PSaT-20, and PSaT-21 were obtained from S. agalactiae and S. iniae cultures isolated from diseased Tilapia (Oreochromis niloticus), and phages PSaG-1, PSaG-2, and PSaG-3 were isolated from diseased Gouramy (Osphoremus gouramy). These phages were previously storaged in a refrigerator for five weeks and were propagated each week in S. agalactiae and $S$. iniae strain using a double-layer agar method. These bacteria were subcultured on BHIA.

Briefly, a mixture of phage and bacteria were cultured in soft agar overlaid BHIA at $28^{\circ} \mathrm{C}$ for 24 hours. After the soft agar showing plaque and having lysis activity, the plaque was cut and collected in BHIB and BHIB enriched with glycerol and kept in refrigerator until testingit for every week. The lytic activities of bacteriophages were observed each week to know its capability against S. agalactiae and S. iniae.

\section{RESULT AND DISCUSSION}

\section{Survival of Phages in Vitro Conditions}

The following experiment was carried out in storage temperature of $8^{\circ} \mathrm{C}$. All phages survived for three weeks in BHIB and BHIB enriched with glycerol. In the fifth week, the survival of phages suddenly decreased except phage PSaT-18. All the phages were differentiated each other based on their lytic activity pattern as shown in Figure 1.

The present in vitro investigation revealed that the $S$. agalactiae phages were tolerant to physico-chemical factors such as storage temperature and types of media. Although they were stable only in three weeks except phage PSaT-18, three weeks survival rate were sufficient in treating Streptococcosis-infected fish. Phage PSaT-18 was a potential candidate for phage therapy because it can live for more than three weeks, although it needs further experiment especially through in vivo condi-
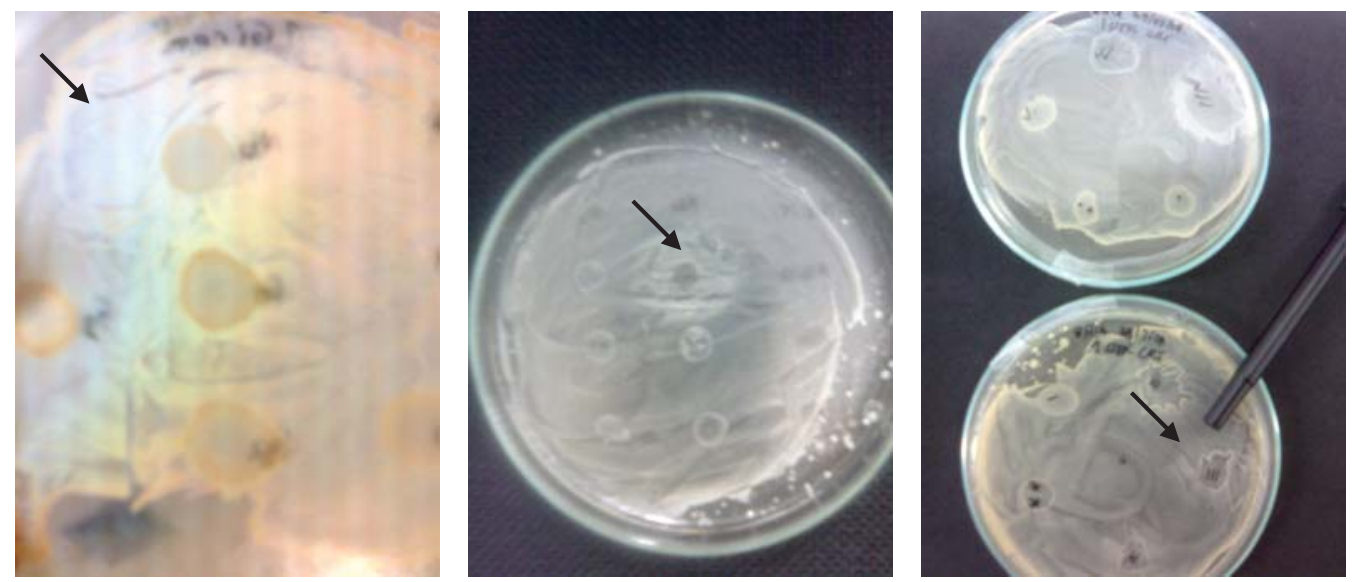

Figure 1. The lytic activity pattern of S. agalactiae bacteriophages 
Table 1. Effect of storage temperature $8^{\circ} \mathrm{C}$ on the lytic activities of phages

\begin{tabular}{ccccccccc}
\hline \multirow{7}{*}{ Weeks } & \multicolumn{7}{c}{ Bacteriophages } \\
\cline { 2 - 8 } & PSaT-18 & PSaT-19 & PSaT-20 & PSaT-21 & PSaG-1 & PSaG-2 & PSaG-3 \\
\hline 1 & ++ & + & + & + & + & + & + \\
2 & ++ & + & + & + & + & + & + \\
3 & +++ & + & + & + & + & + & + \\
4 & ++ & + & + & + & - & - & - \\
5 & + & - & - & - & - & - & - \\
\hline
\end{tabular}

+ : positive, survive ; - : negative, death

tion. The effects of storage temperature of $8^{\circ} \mathrm{C}$ on the lytic activity of phages are shown in Table 1.

According to Park et al. (1997), ideal incubation temperature was $17^{\circ} \mathrm{C}-29^{\circ} \mathrm{C}$ on the lytic activity of phages. In this research, the lytic activity of phage was observed at incubation temperature $28^{\circ} \mathrm{C}$, and all the phages had a positive lytic activity for three weeks. Phages PSaT-18 had higher lytic activity from the first to the third week and decreased on the fourth and the fifth weeks. On the third week, N1 8 had the maximum lytic activity. The other phages showed constant lytic activity and became weak on the fourth and fifth weeks. Because their survive capability average is only three weeks, at present we have no reliable reference experiments for phage therapy or control in the field of fish pathology.

Two types of media (BHIB, BHIB enriched glycerol $1: 1)$ were highly effective for storing $S$. agalactiae phage. But in the fifth week, phages in BHIB enriched glycerol did not have successful growth. After three weeks, almost all phages showed the same lysis pattern. Phage PSaT-18 was very sensitive to any of the tested isolates (S. agalactiae and S. iniae). Bacteriophages can theoretically be a tool for treating infectious disease, but there have been a limited number of reports on their potential use as biological control agents for naturally occuring diseases. Phages of some fish pathogenic bacteria, such as Aeromonas hydrophila (Merino et al., 1990) have been reported but no attempts on phage control were made for bacterial infections in fish. Therefore, further detailed investigations on the phage PSaT-1 8 were needed. Furthermore, the relationship between phage types and pathogenicity or virulence should be investigated and studied the future.

\section{ACKNOWLEDGMENT}

The authors thank the pathology team of the Research Center of Aquaculture Jakarta (Lila Gardenia, Yani Aryati, Tatik Mufidah) for assisting this research.

\section{REFERENCES}

Lorch, A. 1999. Bacteriophages: an alternative to antibiotics? Australasian Biotech. 9(4): 265-269.

Merino, S., S. Camprubi, and J.M. Thomas. 1990. Isolation and characterization of bacteriophage PM2 from Aeromonas hydrophila. FEMS Microbiol Lett. 68: 239-244.

Nakai, T. and S.C. Park. 2002. Bacteriophages therapy of infectious diseases in aquaculture. Res. In Mibrobiol. 153: 13-18.

Park, K.H., S. Matsuoka, T. Nakai, and K. Muroga. 1997. A virulent bacteriophage of Lactococcus garviae isolated from yellowtail Seriola quinqueracliata. Dis. Of Aquat. Org. 29: 145-149.

Plumb, J.A. 1994. Streptococcus and Enterococcus septicaemia. In: Health maintenance of cultured fishes. Principal microbial diseases. CRC Press, Boca Raton, FL. p. $231-238$.

Shoemaker, C.A., P.H. Klasius, and J.J. Evans. 2001. Prevalence of Streptococcus iniae in Tilapia, hybrid striped bass and channel catfish on commercial fish farms in the United States. AJVR. 62: 174-177.

Slopek, S., R. Weber-Dabrowska, M. Dabrowski, and A. Kucharewics-Krukowska. 1987. Results of bacteriophage treatment of suppurative bacterial infection in the years 1981-1986. Arch. Immunol. Ther. Exp. 38: $569-583$. 